\title{
Efecto de la condición hídrica de vegas altoandinas del desierto transicional de Chile sobre algunas características comunitarias de su dipterofauna
}

\author{
Effect of water-condition of high Andean wet pastures of the transitional desert \\ of Chile on some community attributes of their diptero-fauna
}

\author{
Jorge Cepeda-Pizarro ${ }^{*}$, Jaime Pizarro-Araya ${ }^{1}$,Christian R. González ${ }^{2}$
}

\begin{abstract}
RESUMEN
En relación con los invertebrados, el conocimiento acerca de la biota de las vegas altoandinas del norte-centro de Chile es claramente asimétrico. En este trabajo, mediante el empleo de trampas de intercepción de caída y aérea, se estudió la dipterofauna de tres vegas diferentes en su condición hídrica. Del total capturado, la vega húmeda aportó el 52,1\%; la vega seca, el 38,3\% y la vega mésica, el 9,6\%. Se registró la presencia de 29 familias, estimándose un máximo de 30. Por tipo de vega se registraron pequeñas diferencias en el número de familias comunes y exclusivas. Entre vegas, mientras Muscidae dominó numéricamente en la vega seca; Muscidae y Chironomidae codominaron en la vega mésica; a su vez, Chironomidae y Sphaeroceridae lo hicieron en la vega húmeda. Se detectó un efecto de la condición hídrica de la vega sobre las relaciones entre familias de su abundancia relativa. Este efecto se observó igualmente en las comparaciones de diversidad y equitabilidad. El agua está siendo un recurso cada vez más escaso en la región desértica de Chile. Por ello, vegas como las estudiadas en esta ocasión están en un estado muy vulnerable. Este trabajo aporta antecedentes que muestran que no solo los elementos bióticos mayores de estas unidades de paisaje están en situación de riesgo, sino que también los elementos menos conspicuos de ellas, pero no menos importantes por ser la base de muchas de las cadenas alimentarias y los procesos de descomposición de la materia que tienen lugar en el ambiente andino.
\end{abstract}

Palabras clave: Andes semiáridos, biodiversidad, diptera, ecosistemas de montaña, pastizales húmedos, insectos andinos.

\begin{abstract}
Regarding the invertebrates, knowledge about the biota of the Andean peatlands of north-central Chile is clearly asymmetric. In this paper, by using pitfall traps and aerial traps, the diptero-fauna inhabiting three peatlands under different water condition was studied. Of total catch, percent contribution by peatland was as follows: $52.1 \%$ (wet peatland); $38.3 \%$ (dry peatland), and $9.6 \%$ (mesic peatland). The presence of 29 families was recorded, with an estimation of 30 as maximum. Regarding the number of common and exclusive families, small differences between peatlands were recorded. Among peatlands, Muscidae numerically dominated in the dry peatland; Chironomidae and Sphaeroceridae did in the wet peatland; in turn, Muscidae and Chironomidae co-dominated in the mesic one. An effect of water condition of the peatland on the relationships of relative abundance of families was detected. This effect was also observed regarding diversity and evenness. Water is being increasingly scarce in the desert region of Chile. Consequently, peatlands as the studied in this occasion are in a very vulnerable state. This paper provides background showing that not only the larger biotic elements of these landscape units are at risk, but also the smaller ones as well; although not less important for being the base of many food chains and decomposition processes that take place in the arid Andean environment.

Key words: Semiarid Andes, biodiversity, diptera, highland ecosystems, wet pastures, andean insects.
\end{abstract}

\section{Introducción}

Donde las condiciones orográficas y de humedad de los Andes desértico-transicionales de Chile lo permiten, se forman las vegas altoandinas (Squeo et al., 1994, 2006a). Considerando que estas unidades del paisaje andino-desértico son importantes proveedores de servicios (i.e. focos de biodiversidad, almacenamiento de agua y provisión de recursos forrajeros, ecoturismo), que están sujetos a una presión antrópica incremental por los recursos que contienen, y a la acción de

1 Laboratorio de Entomología Ecológica, Depto. de Biología, Facultad de Ciencias, Universidad de La Serena, Casilla 599, La Serena, Chile.

2 Instituto de Entomología, Universidad Metropolitana de Ciencias de la Educación, Casilla 147, Santiago, Chile.

* Autor para correspondencia: jcepeda@userena.cl

Fecha de Recepción: 14 Mayo, 2015.

Fecha de Aceptación: 4 Diciembre, 2015. 
probables efectos del cambio climático global, la base de información y conocimiento referida a estos sistemas naturales ha aumentado durante los últimos años (Sanhueza et al., 2009; Alvial et al., 2012, 2013, 2014; Cepeda-Pizarro et al., 2013; Cepeda-Pizarro \& Armijo, 2014). No obstante, este conocimiento es asimétrico. Abundante en lo concerniente a sus elementos mayores (Squeo et al., 1994, 2006a, 2006b; Osorio et al., 2006; Ahumada \& Faúndez, 2007; Trivelli \& Huerta, 2014), pero reducido en lo concerniente a sus elementos menores, particularmente en lo correspondiente a los grupos de animales invertebrados (Cepeda-Pizarro et al., 2006, 2013). En atención a que en los ecosistemas de montaña la presencia y el éxito colonizador de los invertebrados depende estrechamente de su capacidad de adaptación a las condiciones ambientales imperantes, generalmente extremas (Haslett, 1997; Hinden et al., 2005; Füderer et al., 2006), y que estas condiciones pueden estar cambiando, se hace necesario mejorar el nivel del conocimiento respecto de estos organismos.

Uno de los grupos taxonómicos dominantes del ensamble de invertebrados de los humedales altoandinos del desierto transicional de Chile lo constituyen los dípteros (Cepeda-Pizarro \& Pola, 2013; Cepeda-Pizarro et al., 2013). Aunque se carece de información local, estos insectos desempeñan roles importantes como agentes polinizadores, descomponedores y recursos tróficos importantes para los animales vertebrados en humedales de otras regiones (Keiper et al., 2002; van der Valk, 2006; Beltrán-Concha et al., 2012). En atención a los requerimientos ambientales y los ciclos de vida de muchas de sus especies, diversos autores le otorgan a Diptera la potencialidad de indicador de los cambios que en el humedal pueden estar ocurriendo (Keiper et al., 2002; Sanford et al., 2003). Considerando que la condición hídrica del humedal juega un papel importante en la presencia y en los ciclos de vida de diversas especies de invertebrados (Lods-Crozet \& Castella, 2009), en este trabajo se estableció como hipótesis de trabajo que la condición hídrica de las vegas de los Andes desértico-transicionales de Chile influye en las características del ensamble de Diptera. Los objetivos del trabajo fueron i) documentar, a nivel de familia, la composición de la díptero-fauna presente en tres vegas altoandinas localizadas en una cuenca hidrográfica del desierto transicional de Chile; ii) describir, a nivel de familia, el ensamble de Diptera en términos de los parámetros de riqueza (RF), diversidad (DF) y equitabilidad (EF), iii) evaluar el efecto de la condición hídrica de la vega sobre las relaciones de abundancia y los parámetros comunitarios arriba indicados, e iv) identificar la contribución relativa de las familias a las diferencias observadas entre ensambles, según la condición hídrica de la vega.

\section{Materiales y Métodos}

\section{Ubicación y vegas estudiadas}

Como casos de estudio se seleccionaron tres vegas. Estas forman parte de la subcuenca del río del Toro ( 430 km²; 2520-4250 msnm; Zavala, 2006), en el piso altoandino de la hoya hidrográfica del río Elqui (Región de Coquimbo, Chile) (Figura 1). Los criterios usados en su selección fueron: 1) tamaño similar; 2) posición altitudinal cercana; 3) un adecuado conocimiento de su flora dominante; 4) diferente condición hídrica, evaluada por examen visual y presión manual a muestras del prado obtenidas mediante calicatas para suelo, y 5) presencia/ausencia de cuerpos de agua corriente y estancada. Las vegas seleccionadas fueron Las Mulas (VLM, 1 ha de superficie), ubicada en la quebrada de Las Mulas (3100 msnm); Pastos Largos (VPL, 1-2 ha), en la quebrada de Pastos Largos (3600 msnm) y vega Tambo (VTB, 2 h), en la quebrada del estero Tambo (3950 msnm) (Figura 1). Detalles del área de estudio se encuentran en Cepeda-Pizarro et al. (2006). De acuerdo con las características hídricas y vegetacionales, VLM correspondió a una vega de tipo seco; VPL, tipo mésico y VTB, tipo húmedo. Descripciones de estas vegas se encuentran en Cepeda-Pizarro \& Pola (2013).

\section{Análisis del ensamble de Diptera}

Los especímenes fueron capturados con trampas Barber (intercepción de caída) y Malaise (intercepción área). Se realizaron tres campañas mensuales de muestreo durante la estación de crecimiento documentada para el área (Squeo et al., 2006a, 2006b), según la secuencia diciembre/enero a febrero/ marzo. Las comparaciones realizadas se basaron en las sumas de los aportes de cada tipo de trampa. Los detalles metodológicos empleados se describen en Cepeda-Pizarro \& Pola (2013) y Cepeda-Pizarro et al. (2013). En la identificación taxonómica del material capturado se usó la colección de referencia 


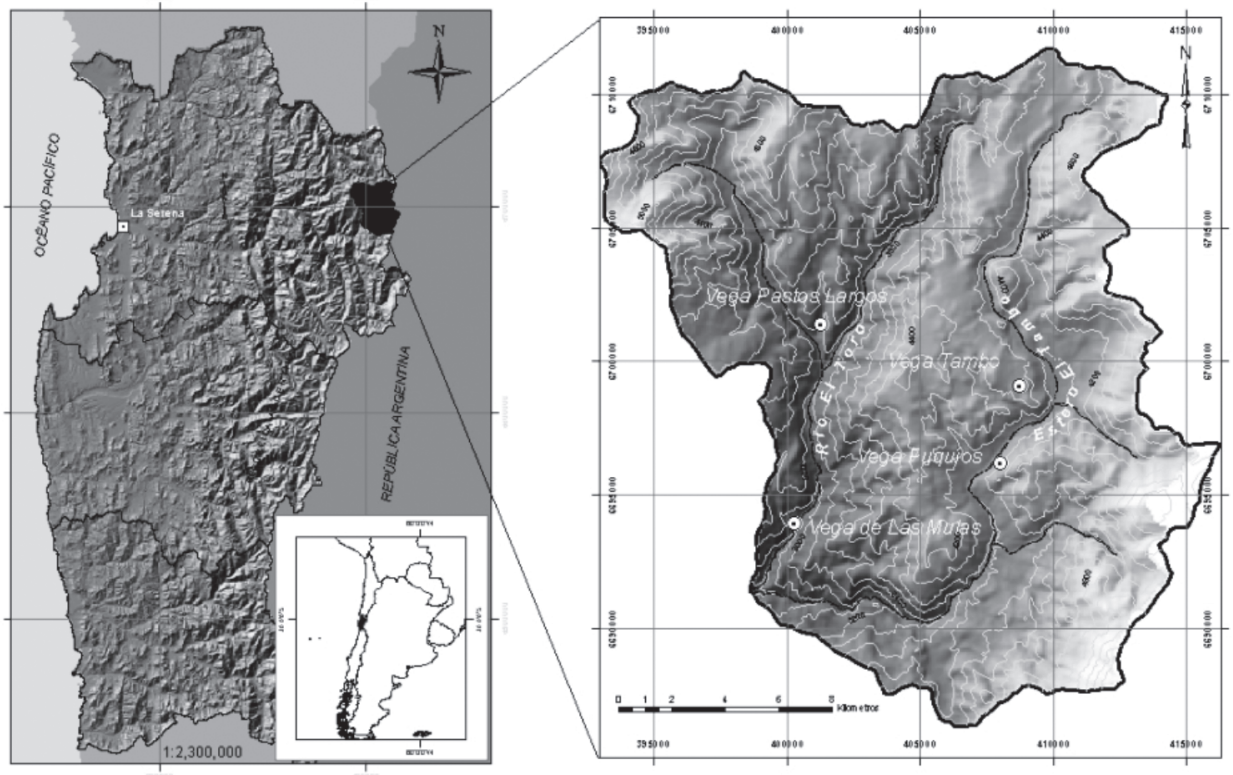

Figura 1. Ubicación geográfica del área de estudio y vegas estudiadas; subcuenca del río del Toro; piso altoandino de la hoya hidrográfica del río Elqui (Región de Coquimbo, Chile).

depositada en el Instituto de Entomología de la Universidad Metropolitana. Siguiendo a Timms et al. (2014) y al grado del conocimiento taxonómico actualmente disponible para este taxón en Chile, la categoría usada como referencia fue familia, según McAlpine et al. (1981, 1987).

El diseño usado en el estudio fue seudoexperimental, con la condición hídrica de la vega como la variable seudoexperimental (factor fijo: condición hídrica $=3$ ) y mes de muestreo como réplica (mes de muestreo=3). La evaluación del efecto de la condición hídrica de la vega sobre las relaciones de abundancia relativa entre familias se realizó con la prueba PERMANOVA y t-pareada (Anderson, 2005). El análisis PERMANOVA se basó en el índice Bray-Curtis de disimilitud (disimilaridad), con los datos originales $(x+1)$ transformados logarítmicamente (ln). La RF fue estimada según los estimadores Chao y Lee 1 (ACE), Jackknife 1 y Rarefacción (agrupada, finita); la DF fue estimada según los índices Simpson (D), Brillouin (D), y dominancia (Berger-Parker): la EF fue estimada mediante los índices Simpson E, Camargo, Smith y Wilson B y NHC. Los cálculos fueron realizados con el programa "Species Diversity and Richness IV" (Seaby \& Henderson, 2006). Complementariamente, los resultados se compararon mediante las pruebas ANOSIM y SIMPER sobre los datos originales sin transformar. Para este último propósito los cálculos fueron realizados con el programa CAP (Henderson \& Seaby, 2008).

\section{Resultados}

\section{Ensamble de Diptera}

Se capturaron 29.160 especímenes de Diptera. De estos, el 38,3\% fue aportado por VLM (condición vega seca), seguida por VTB $(-52 \%)$ (condición vega húmeda) y VPL ( 10\%) (condición vega mésica). Se registró la presencia de 29 familias, ocho de ellas con abundancia numérica relativa total $\leq 0,1 \%$. Debido a este nivel de abundancia y en el contexto de este trabajo, estas familias se consideraron escasas o raras en el sitio de estudio. Este número varió ligeramente entre vegas (Tabla 1). Considerando a las familias con aporte $\geq 10 \%$ del gran total, cuatro familias dominaron numéricamente el ensamble: Muscidae $(31,3 \%)$, Chironomidae $(15,7 \%)$, Sphaeroceridae $(13,7 \%)$ y Heleomyzidae $(10,1 \%)$. Entre vegas, mientras Muscidae dominó numéricamente en VLM (24,3\% del gran total); Muscidae y Chironomidae codominaron en VPL $(2,9 \%)$, y Chironomidae $(11,2 \%)$ y Sphaeroceridae $(13,6 \%)$ lo hicieron en VTB (Tabla 1). Se registraron pequeñas diferencias en el número de familias comunes y exclusivas por 
Tabla 1. Abundancia relativa de familias de Diptera registradas en tres vegas altoandinas de una hoya hidrográfica del desierto transicional de Chile.

\begin{tabular}{|c|c|c|c|c|c|c|c|c|}
\hline \multirow[b]{3}{*}{ Familias $^{1}$} & \multicolumn{6}{|c|}{ Vega } & \multirow{2}{*}{\multicolumn{2}{|c|}{ Gran total }} \\
\hline & \multicolumn{2}{|c|}{ VLM } & \multicolumn{2}{|c|}{ VPL } & \multicolumn{2}{|c|}{ VTB } & & \\
\hline & $\mathrm{n}$ & $\%$ & $\mathrm{n}$ & $\%$ & $\mathrm{n}$ & $\%$ & $\mathrm{n}$ & $\%$ \\
\hline Agromyzidae & 67 & 0,2 & 39 & 0,1 & 0 & 0 & 106 & 0,4 \\
\hline Asilidae & 0 & 0,0 & 1 & $<0,1$ & 0 & 0 & 1 & $<0,1$ \\
\hline Bibionidae & 9 & 0,0 & 0,0 & 0,0 & 0 & 0 & 9 & $<0,1$ \\
\hline Bombyliidae & 109 & 0,4 & 51 & 0,2 & 180 & 0,6 & 340 & 1,2 \\
\hline Calliphoridae & 0 & 0,0 & 21 & 0,1 & 37 & 0,1 & 58 & 0,2 \\
\hline Ceratopogonidae & 29 & 0,1 & 33 & 0,1 & 116 & 0,4 & 178 & 0,6 \\
\hline Chironomidae & 466 & 1,6 & 835 & 2,9 & 3264 & 11,2 & 4565 & 15,7 \\
\hline Chloropidae & 0 & 0,0 & 1 & $<0,1$ & 197 & 0,7 & 198 & 0,7 \\
\hline Culicidae & 0 & 0,0 & 33 & 0,1 & 0 & 0,0 & 33 & 0,1 \\
\hline Dolichopodidae & 402 & 1,4 & 60 & 0,2 & 246 & 0,8 & 708 & 2,4 \\
\hline Empididae & 91 & 0,3 & 235 & 0,8 & 1373 & 4,7 & 1699 & 5,8 \\
\hline Ephydridae & 1 & $<0,1$ & 0 & 0,0 & 1106 & 3,8 & 1107 & 3,8 \\
\hline Heleomyzidae & 845 & 2,9 & 0 & 0,0 & 2114 & 7,2 & 2959 & 10,1 \\
\hline Muscidae & 7097 & 24,3 & 834 & 2,9 & 1197 & 4,1 & 9128 & 31,3 \\
\hline Mycetophilidae & 0 & 0,0 & 0 & 0,0 & 113 & 0,4 & 113 & 0,4 \\
\hline Nemestrinidae & 1 & $<0,1$ & 0 & 0,0 & 0 & 0 & 1 & $<0,1$ \\
\hline Ulidiidae & 12 & $<0,1$ & 0 & 0,0 & 0 & 0 & 12 & $<0,1$ \\
\hline Phoridae & 299 & 1,0 & 61 & 0,2 & 215 & 0,7 & 575 & 2,0 \\
\hline Psychodidae & 6 & $<0,1$ & 0 & 0,0 & 2 & $<0,1$ & 8 & $<0,1$ \\
\hline Sarcophagidae & 206 & 0,7 & 29 & 0,1 & 2 & $<0,1$ & 237 & 0,8 \\
\hline Sciaridae & 52 & 0,2 & 43 & 0,1 & 178 & 0,6 & 273 & 0,9 \\
\hline Simuliidae & 77 & 0,3 & 82 & 0,3 & 121 & 0,4 & 280 & 1,0 \\
\hline Sphaeroceridae & 0 & 0,0 & 32 & 0,1 & 3955 & 13,6 & 3987 & 13,7 \\
\hline Stratiomydae & 167 & 0,6 & 69 & 0,2 & 0 & 0 & 236 & 0,8 \\
\hline Syrphidae & 699 & 2,4 & 195 & 0,7 & 602 & 2,1 & 1496 & 5,1 \\
\hline Tabanidae & 15 & $<0,1$ & 2 & $<0,1$ & 19 & 0,1 & 36 & 0,1 \\
\hline Tachinidae & 521 & 1,8 & 121 & 0,4 & 55 & 0,2 & 697 & 2,4 \\
\hline Therevidae & 0 & 0,0 & 14 & $<0,1$ & 46 & 0,2 & 60 & 0,2 \\
\hline Tipulidae & 3 & $<0,1$ & 6 & $<0,1$ & 30 & 0,1 & 39 & 0,1 \\
\hline Otras & 0,00 & 0,0 & 0 & 0,0 & 21 & 0,1 & 21 & 0,1 \\
\hline Gran total- $\%$ & 11174 & 38,3 & 2797 & 9,6 & 15189 & 52,1 & 29160 & 100,0 \\
\hline Total F Observado & & 21,0 & & 22,0 & & 22,0 & & 29,0 \\
\hline Familias $>0,1 \%$ & & 14,0 & & 10,0 & & 17,0 & & 21,0 \\
\hline
\end{tabular}

${ }^{1}$ Especímenes capturados mediante trampeos Barber y Malaise realizados durante la estación de crecimiento de la vega. Código para vegas: VLM: vega Las Mulas (vega seca); VPL: vega Pastos Largos (vega mésica); VTB: vega Tambo (vega húmeda).

tipo de vega (Tablas 2-4). El número predicho para el total de familias varió entre 29 (Chao y Lee 1; rarefacción) y 31 (jackknife 1).

\section{Efecto sobre la condición hídrica de la vega sobre las características del ensamble}

No se observó tendencia respecto del número estimado de familias por tipo de vega. Comparado con el número observado de familias por vega, el método jackknife 1 aparentemente sobrestimó el número esperado de familias, especialmente para el caso de la vega húmeda. Por el contrario, los valores entregados por los métodos rarefacción (agrupada, finita) y ACE resultaron más cercanos a los observados, especialmente con el primero de ellos (Tabla 5). La prueba PERMANOVA mostró un efecto de la condición hídrica de la vega sobre las relaciones de abundancia entre familias $\left(\mathrm{p}_{\text {perm. } 2 ; 6}=0,0078 ; \mathrm{p}_{\mathrm{MC} .2 ; 6}=0,001\right)$. Dos de las tres comparaciones pareadas mostraron diferencias significativas; aunque la tercera arrojó un valor-t cercano al crítico $(0,05 \%)$. Las comparaciones que resultaron significativas fueron vega seca/vega mésica y vega seca/vega húmeda, con una estrecha diferencia entre ambas respecto del índice de disimilaridad (e.g. 31,2-32,1) (Tablas 6 y 7). En este trabajo se usaron tanto índices que enfatizan en los cálculos 
Tabla 2. Distribución de familias de Diptera según la condición hídrica de la vega: comparación vega seca (VLM)-vega mésica (VPL).*

\begin{tabular}{|c|c|c|}
\hline $\begin{array}{c}\text { Exclusivas a VLM } \\
\text { (6) }\end{array}$ & $\begin{array}{c}\text { Comunes } \\
\text { (17) }\end{array}$ & $\begin{array}{c}\text { Exclusivas a VPL } \\
\text { (6) }\end{array}$ \\
\hline $\begin{array}{l}\text { Bibionidae } \\
\text { Ephydridae } \\
\text { Heleomyzidae } \\
\text { Nemestrinidae } \\
\text { Ulidiidae } \\
\text { Psychodidae }\end{array}$ & $\begin{array}{l}\text { Agromyzidae } \\
\text { Bombyliidae } \\
\text { Ceratopogonidae } \\
\text { Chironomidae } \\
\text { Dolichopodidae } \\
\text { Empididae } \\
\text { Muscidae } \\
\text { Mycetophilidae } \\
\text { Phoridae } \\
\text { Sarcophagidae } \\
\text { Sciaridae } \\
\text { Simuliidae } \\
\text { Stratiomydae } \\
\text { Syrphidae } \\
\text { Tabanidae } \\
\text { Tachinidae } \\
\text { Tipulidae }\end{array}$ & $\begin{array}{l}\text { Asilidae } \\
\text { Calliphoridae } \\
\text { Chloropidae } \\
\text { Culicidae } \\
\text { Sphaeroceridae } \\
\text { Therevidae }\end{array}$ \\
\hline
\end{tabular}

*Código para vega: como en Tabla 1.

Tabla 3. Distribución de familias de Diptera según la condición hídrica de la vega: comparación vega seca (VLM)-vega húmeda (VTB).*

\begin{tabular}{|c|c|c|}
\hline $\begin{array}{c}\text { Exclusivas a VLM } \\
\text { (5) }\end{array}$ & $\begin{array}{c}\text { Comunes } \\
\text { (19) }\end{array}$ & $\begin{array}{c}\text { Exclusivas a VTB } \\
\text { (5) }\end{array}$ \\
\hline $\begin{array}{l}\text { Agromyzidae } \\
\text { Bibionidae } \\
\text { Nemestrinidae } \\
\text { Ulidiidae } \\
\text { Stratiomydae }\end{array}$ & $\begin{array}{l}\text { Asilidae } \\
\text { Culicidae } \\
\text { Ephydridae } \\
\text { Heleomyzidae } \\
\text { Bombyliidae } \\
\text { Ceratopogonidae } \\
\text { Chironomidae } \\
\text { Dolichopodidae } \\
\text { Empididae } \\
\text { Muscidae } \\
\text { Phoridae } \\
\text { Psychodidae } \\
\text { Sarcophagidae } \\
\text { Sciaridae } \\
\text { Simuliidae } \\
\text { Syrphidae } \\
\text { Tabanidae } \\
\text { Tachinidae } \\
\text { Tipulidae }\end{array}$ & $\begin{array}{l}\text { Calliphoridae } \\
\text { Chloropidae } \\
\text { Mycetophilidae } \\
\text { Sphaeroceridae } \\
\text { Therevidae }\end{array}$ \\
\hline
\end{tabular}

*Código para vega como en Tabla 1.

los grupos escasos (raros) (Brillouin D) como índices que enfatizan los grupos numéricamente dominantes (Simpsons D; dominancia de B-P). Para las tres vegas de la subcuenca, la DF (valor
Tabla 4. Distribución de familias de Diptera según la condición hídrica de la vega: comparación vega mésica (VPL)-vega húmeda (VTB).*

\begin{tabular}{|c|c|c|}
\hline $\begin{array}{c}\text { Exclusivas a VPL } \\
\text { (4) }\end{array}$ & $\begin{array}{c}\text { Comunes } \\
\text { (21) }\end{array}$ & $\begin{array}{l}\text { VTB } \\
(4)\end{array}$ \\
\hline $\begin{array}{l}\text { Agromyzidae } \\
\text { Asilidae } \\
\text { Culicidae } \\
\text { Stratiomydae }\end{array}$ & $\begin{array}{l}\text { Calliphoridae } \\
\text { Chloropidae } \\
\text { Heleomyzidae } \\
\text { Bombyliidae } \\
\text { Ceratopogonidae } \\
\text { Chironomidae } \\
\text { Dolichopodidae } \\
\text { Empididae } \\
\text { Muscidae } \\
\text { Nemestrinidae } \\
\text { Phoridae } \\
\text { Sarcophagidae } \\
\text { Sciaridae } \\
\text { Sphaeroceridae } \\
\text { Simuliidae } \\
\text { Syrphidae } \\
\text { Tabanidae } \\
\text { Tachinidae } \\
\text { Therevidae } \\
\text { Ulidiidae } \\
\text { Tipulidae }\end{array}$ & $\begin{array}{l}\text { Ephydridae } \\
\text { Heleomyzidae } \\
\text { Mycetophilidae } \\
\text { Psychodidae }\end{array}$ \\
\hline
\end{tabular}

*Código para vega como en Tabla 1.

puntual \pm ee) fluctuó entre 2,23 $\pm 0,12$ (Brillouin D) y 6,2 $\pm 0,91$ (Simpsons D). A nivel de vega, ambos índices fueron consistentes en mostrar una tendencia positiva con el aumento de humedad. Complementariamente, el índice de dominancia $\mathrm{B}-\mathrm{P}\left(\mathrm{n}_{\max } / \mathrm{N}\right)$ mostró la tendencia opuesta (Figura 2). Los valores estimados de EF para toda la subcuenca fluctuaron (valor puntual \pm ee) entre $0,98 \pm 0,05$ (índice de Camargo) y 0,08 $\pm 0,03$ (índice NHC). A nivel de vega, mientras Simpson E tendió a aumentar a mejor condición hídrica $(0,16$ a 0,30$)$; los índices restantes no evidenciaron alguna tendencia (Figura 3 ). Las comparaciones pareadas mediante boostraping de la DF mostraron que las diferencias observadas fueron significativas con los tres índices usados; por el contrario, las comparaciones pareadas de la EF entregaron resultados inconsistentes (Tabla 8). La prueba ANOSIM mostró diferencias significativas entre ensambles según la condición hídrica de la $\operatorname{vega}\left(R_{\text {global }}=0,82 ; p \leq 0,001 ;\right.$ nivel $\left.\leq 0,1 \%\right)$. La prueba SIMPER entregó valores globales de disimilaridad entre vegas relativamente altos $(65,19-73.70 \%)$. En la Tabla 9 se muestra el comportamiento por vega de la abundancia promedio de las familias con mayor contribución porcentual a la explicación 


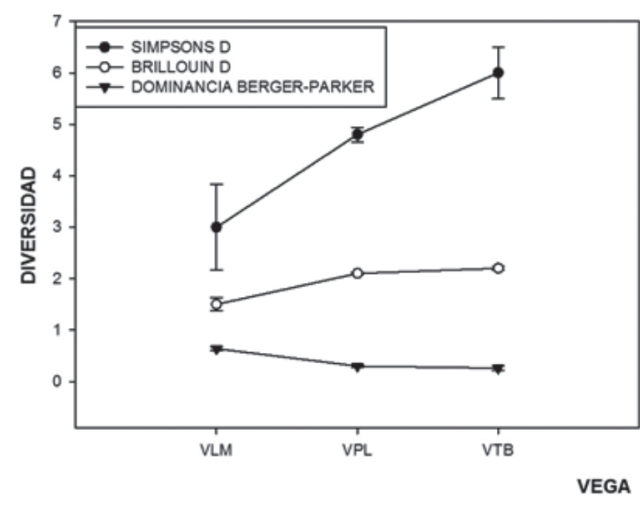

Figura 2. Valores de los índices de diversidad Simpsons' D, Brillouin D y dominancia de Berger-Parker, según la condición hídrica de la vega. Código para vega: como en Tabla 1. La barra vertical corresponde al error estándar estimado mediante bootstraping.

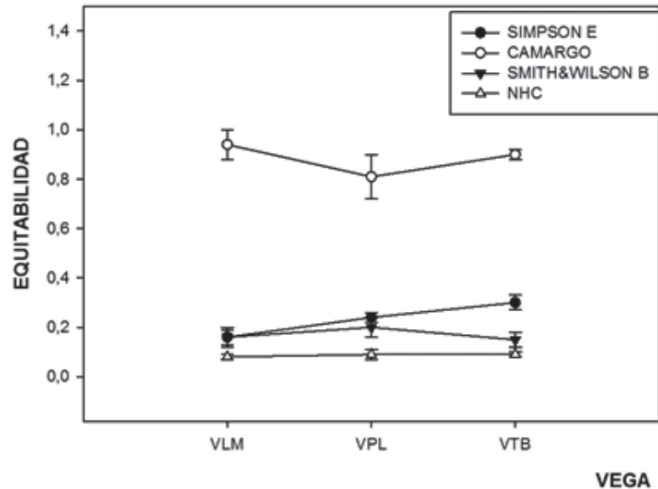

Figura 3. Valores de los índices de equitabilidad Simpsons' E, Camargo, Smith y Wilson B y NHC, según la condición hídrica de la vega. Código para vega y significado de la barra vertical como en Figura 2.

Tabla 5. Riqueza estimada de familias (RF) del ensamble de Diptera registrado en tres vegas altoandinas del desierto transicional de Chile (entre paréntesis: * (de); ** (ee)).

\begin{tabular}{lcccc}
\hline \multirow{2}{*}{ Estimador } & \multicolumn{3}{c}{ Vega } \\
\cline { 2 - 5 } & VLM & VPL & VTB & Total FR \\
\hline Chao y Lee 1 (ACE) & 20,01 & 22,03 & 21,5 & 29 \\
Jackknifing 1* & $24,8(6,4)$ & $31,5(12,5)$ & $33,04(12,3)$ & $31(1,33)$ \\
Rarefacción (agrupada, finita)** & $22(<0,01)$ & $22(<0,01)$ & $22(<0,01)$ & $29(<0,08)$ \\
N $^{\circ}$ F observadas & 21 & 22 & 22 & 29 \\
$\mathrm{~N}^{\circ} \mathrm{F}>0,1 \%$ & 14 & 10 & 17 & 22 \\
\hline
\end{tabular}

Código para vega como en Tabla 1.

Tabla 6. Evaluación del efecto de la condición hídrica de la vega sobre las relaciones de abundancia de las familias constituyentes del ensamble de Diptera presente en tres vegas altoandinas del desierto transicional de Chile*.

\begin{tabular}{lcccccc}
\hline FV & GL & SC & MC & F & P (perm) & P (MC) \\
\hline Condición hídrica & 2 & 2088,7 & 1044,4 & 8,5 & 0,0078 & 0,001 \\
Residual & 6 & 739,3 & 123,2 & & & \\
Total & 8 & 2828 & & & & \\
\hline
\end{tabular}

*Prueba PERMANOVA sobre datos logarítmicamente transformados $(\ln (\mathrm{x}+1))$. Valores aproximados a la décima más cercana, excepto valores $P$.

Tabla 7. PERMANOVA. Prueba de t para comparaciones entre pares de ensambles de Diptera, según la condición hídrica de la vega e índice de disimilaridad B-C.

\begin{tabular}{lllll}
\hline Comparación & $\mathrm{t}$ & P_perm & P_MC & B-C \\
\hline VLM/VPL & 1,8 & 0,189 & 0,0716 & 22,6 \\
VLM/VTB & 3,7 & 0,1054 & 0,0038 & 32,1 \\
VPL/VTB & 3,3 & 0,1052 & 0,0082 & 31,2 \\
\hline
\end{tabular}

Valores aproximados a la décima más cercana, excepto valores $P$. 
Tabla 8. Comparación de la diversidad (DF) y la equitabilidad (EF) del ensamble de Diptera según la condición hídrica de la vega.

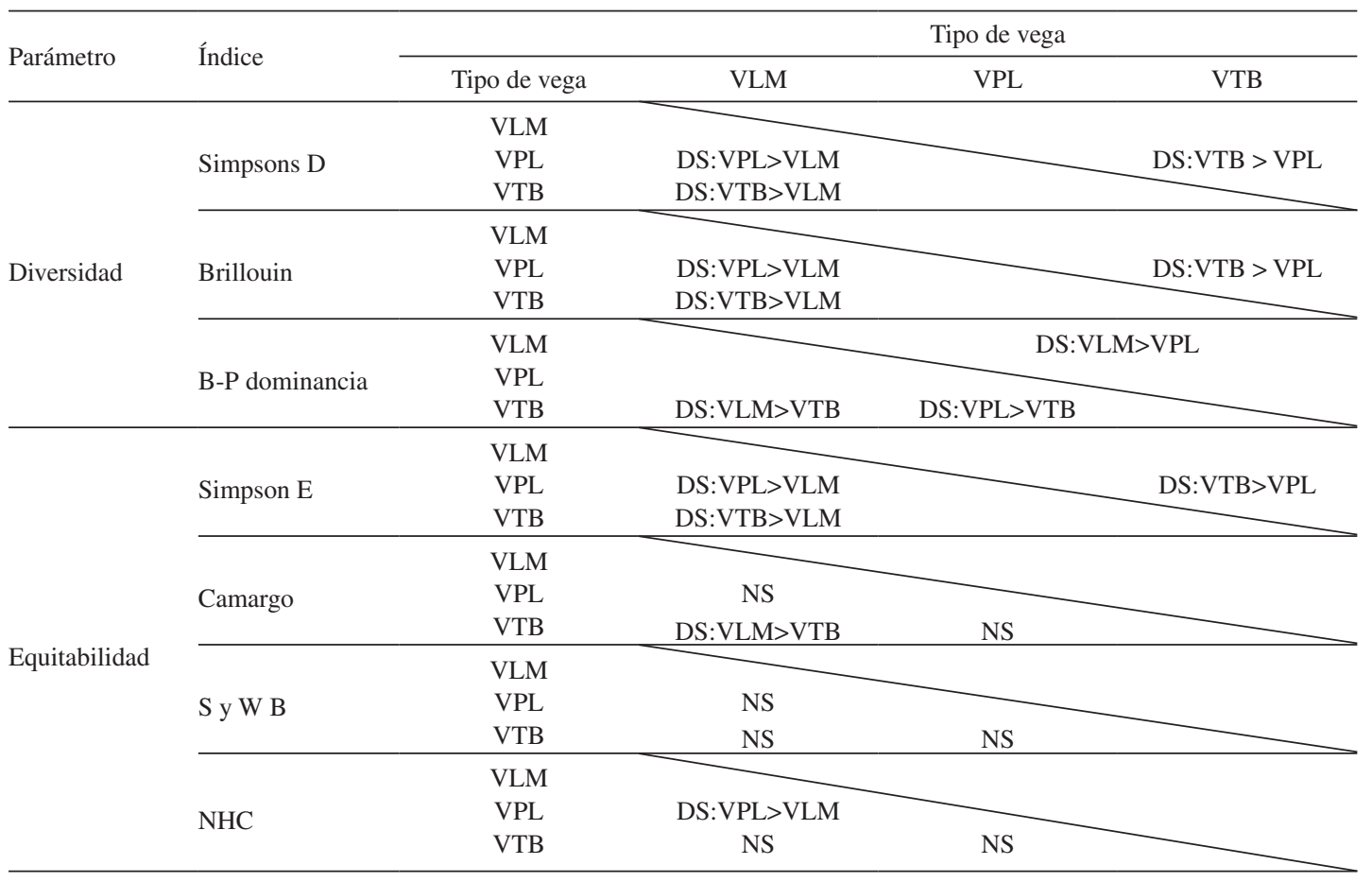

*DS: diferencia significativa al 5\%; NS: comparación no significativa.

Tabla 9. Abundancia promedio de las familias numéricamente dominantes del ensamble de Diptera según la condición hídrica de la vega y valores del índice del disimilaridad entre ensambles (valor promedio y por familias)*.

\begin{tabular}{|c|c|c|c|c|c|c|}
\hline \multirow[b]{2}{*}{ Familia/Comparación } & \multicolumn{3}{|c|}{ Abundancia promedio } & \multicolumn{3}{|c|}{ Disimilaridad entre ensambles } \\
\hline & VLM & VPL & VTB & $\begin{array}{c}\text { VLM/VPL } \\
65,19\end{array}$ & $\begin{array}{c}\text { VLM/TBO } \\
73,70\end{array}$ & $\begin{array}{c}\text { VPL/VTB } \\
72,22\end{array}$ \\
\hline Muscidae & 2365,70 & 278,00 & 399,00 & $37,5(57,56)$ & $20,98(28,47)$ & $2,70(3,71)$ \\
\hline Syrphidae & 233,00 & 65,00 & 200,70 & $4,61(7,10)$ & $1,76(2,42)$ & $2,17(3,00)$ \\
\hline Chironomidae & 155,30 & 278,30 & 1088,00 & $4,41(6,78)$ & $10,91(14,81)$ & $13,05(18,07)$ \\
\hline Dolichopodidae & 134,00 & 20,00 & 82,00 & $2,34(3,60)$ & - & $1,28(1,80)$ \\
\hline Tachinidae & 173,70 & 40,30 & 18,30 & $2,25(3,47)$ & $1,63(2,20$ & - \\
\hline Phoridae & 99,70 & 20,30 & 71,70 & $1,86(2,86)$ & - & - \\
\hline Sarcophagidae & 68,70 & 9,70 & $<0,1$ & $1,83(2,82)$ & - & - \\
\hline Heleomyzidae & 281,70 & 0,00 & 704,70 & $4,48(6,88)$ & $6,17(8,39)$ & $11,72(16,23)$ \\
\hline Simuliidae & 25,70 & 27,30 & 40,30 & - & - & - \\
\hline Stratiomydae & 55,70 & 23,00 & 0,00 & - & - & - \\
\hline Culicidae & 0,00 & 11,00 & 0,00 & - & - & - \\
\hline Sphaeroceridae & 0,00 & 10,70 & 1318,30 & - & $16,15(21,92)$ & $22,73(31,47)$ \\
\hline Empididae & 30,30 & 78,30 & 457,70 & - & $4,84(6,56)$ & $5,79(8,01)$ \\
\hline Ephydridae & $<0,1$ & 0,00 & 368,70 & - & $4,21(5,71)$ & $5,86(8,11)$ \\
\hline
\end{tabular}

*Prueba SIMPER de una vía para la composición numérica del ensamble sobre los datos logarítmicamente transformados (log $(\mathrm{n}+1)$ ). Valores bajo la comparación corresponden a la disimilaridad promedio. En paréntesis, la contribución porcentual por familia a las diferencias observadas entre ensambles. 
de las diferencias observadas entre ensambles. En general, ocho familias fueron los elementos que aportaron el mayor porcentaje de las diferencias. En la comparación vega seca/vega mésica la mayor contribución fue aportada por Muscidae (57,56\%); en la comparación vega seca/vega húmeda este aporte correspondió tanto a Muscidae $(28,47 \%)$ como a Sphaeroceridae $(21,92 \%)$; en la comparación vega mésica/vega húmeda el principal aporte provino de Sphaeroceridae $(31,47 \%)$.

\section{Discusión}

A pesar de la diversidad de humedales que el territorio chileno tiene, la literatura nacional reporta escasos trabajos sobre la entomofauna de estos ambientes. Actúan a favor de esta situación las dificultades derivadas del procesamiento del alto número de ejemplares, generalmente pequeños, que se capturan en este tipo de trabajo. Por ejemplo, en este estudio se capturaron 29.160 individuos, lo que significó un arduo trabajo de separación, diagnóstico inicial a nivel de morfoespecie, recuento y reconocimiento final al nivel taxonómico de familia. Aunque en este trabajo seguimos el concepto de "reemplazante" ("subrogate") para inferir la información subyacente a familia, el estudio de los dípteros incluso a este nivel de resolución taxonómica requiere, en muchos casos, una experticia poco desarrollada en el país. Por su parte, las pruebas de hipótesis se ven obstaculizadas por las exigencias de los métodos estadísticos que demandan tamaños de muestras muchas veces difíciles de satisfacer por costo, tiempo de procesamiento, riesgos de pseudorreplicación y aumento del efecto de la heterogeneidad espacial del sistema bajo análisis, particularmente importante en el caso de la montaña andina. Aun cuando en este trabajo usamos métodos no paramétricos para las pruebas de hipótesis, el tamaño de muestra puede todavía parecer reducido $(r=3)$. Teniendo presente estas condiciones de estudio, algunas ideas han emergido de él.

Para humedales chilenos, la riqueza-F de Diptera se reporta entre cinco y 30 (Hermosilla et al., 1975; Villagrán-Mella et al., 2006; Figueroa et al., 2007; 2009; Cepeda-Pizarro et al., 2013). En este trabajo se registró la presencia de 29 familias, un número ligeramente mayor al encontrado en humedales andinos tropicales (23) (Sánchez \& Amat-García, 2005). Aunque no estudiado, posiblemente el valor de riqueza-F encontrado en este trabajo se debe a que las vegas ofrecen un conjunto diverso de microhábitats y recursos alimentarios en abundancia para su colonización y explotación, como ha sido descrita para humedales de otras latitudes (Acosta \& Prat, 2010; Scheibler et al., 2014). Por otra parte, las diferencias encontradas en la riqueza-F entre las vegas de este estudio y aquellas reportadas en la literatura chilena, probablemente reflejan las diferencias en intervención humana. En otras palabras, el relativamente alto número de familias encontrado en este estudio puede deberse al mayor grado de "pristinidad" que tienen los humedales andinos del área de estudio respecto de aquel de los humedales costeros estudiados por los autores arriba mencionados.

Consistente con su condición de prado húmedo, se registraron familias tanto terrestres como acuáticas y semi-acuáticas. En el anexo Tabla 1 se entregan algunos antecedentes de historia natural de las familias registradas. La presencia de familias con requerimientos terrestres puede interpretarse como una muestra de la influencia del ambiente terrestre circundante, especialmente en el caso de las dos más pequeñas (e.g. VLM y VPL). Considerando a las familias con abundancia $\geq 10 \%$ del gran total, el ensamble estuvo numéricamente dominado por cuatro familias, que representaron el $\sim 71 \%$ (Tabla 1). Muscidae fue la familia más abundante ( 31\%). Según Keiper et al. (2002), relativamente pocas especies de Muscidae viven en ambientes semiacuáticos. Como se ha señalado anteriormente, debido al nivel de resolución taxonómica empleado, no es posible informar en este trabajo si las especies de Muscidae representan movimientos desde el entorno circundante o corresponden a especies propias del humedal. La segunda familia numéricamente dominante correspondió a Chironomidae. En humedales, Chironomidae es la familia de Diptera más ampliamente distribuida y son habitantes conspicuos de muchos ecosistemas acuáticos de montaña (Boggero et al., 2006). Junto a Simuliidae, las moscas quironómidos figuran entre los primeros colonizadores de ríos mediterráneos chilenos (Figueroa et al., 2006). Sphaeroceridae fue la tercera familia numéricamente dominante; esta es una gran familia cosmopolita; sus larvas son saprófagas; diversas especies viven en turberas de montaña; en estos ambientes, las moscas esfarocéridas viven y se alimentan de materia orgánica animal y vegetal en descomposición (Keiper et al., 2002). La cuarta familia en orden 
de importancia numérica fue Heleomyzidae; estas son moscas de hábitos terrestres que se alimentan de materia orgánica vegetal y animal en descomposición. Familias con representaciones numéricas menores fueron Empididae, Syrphidae, Dolichopididae y Tachinidae (Tabla 1). Según la información disponible, Empididae estaría representada en el área principalmente por el género Apalocnemis; Syrphidae, por los géneros Dolichogyna, Melanostoma y Scaeva; Tachinidae, por Prosopochaeta caliginosa Cortes y Campos, 1971, Steatosoma rufiventris Aldrich, 1934, Steatosoma nigriventris Aldrich, 1934, Chaetocnephalia americana (Schiner, 1868), Incamyia spinicosta Aldrich, 1928 y Ateloglutus chilensis (Brethes, 1920). La abundancia relativa de Ephydridae en las vegas fue menor a la esperada según reportes de trabajos realizados en ambientes templados (e.g. Keiper et al., 2002). Adicionalmente, con abundancia relativa baja se registraron otras familias indicadas como comunes y abundantes en humedales de climas templados (Keiper et al., 2002). En esta condición se encuentran Agromyzidae, Chloropidae y Sarcophagidae. Aunque con presencia numérica baja, se registró la presencia de Tabanidae y Simuliidae; las larvas de las moscas tabánidos son habitantes comunes de turberas boreales y subárticas (McElligott \& Lewis, 1996). En el área, Tabanidae está principalmente representada por el género Dasybasis; las larvas de especies de este género usan las vegas como hábitat donde desarrollarse (González, 2002, 2004, 2014). Aunque no registradas en las vegas estudiadas, las moscas simúlidos están bien representadas en humedales andinos (Grillet \& Barrera, 1997). Otras familias registradas en este estudio, aunque en bajo número, fueron Bombyliidae, probablemente representada por los géneros Dischistus, Anthrax (Anthrax squalidus Philippi, 1865) y Villa sp; Ulidiidae, por el género Ceroxys (Ceroxys edwardsi (Malloch, 1933); mientras Stratiomyidae estaría representada en las vegas estudiadas por el género Beridops (Beridops maculipennis Blanchard, 1852).

Resulta evidente que, para una colonización exitosa del ambiente andino, los dípteros deben disponer de las estrategias requeridas para superar las exigencias que este impone. Por ejemplo, estudios realizados en transectos altitudinales (Wissinger et al., 2003) sugieren que la variabilidad propia de los ambientes andinos podría desempeñar un papel importante en la determinación de los patrones de colonización, abundancia y estructura del ensamble de invertebrados, entre ellos, los dípteros. Algunos autores (Batzer \& Wissinger, 1996; Keiper et al., 2002) señalan que para una adecuada interpretación de estos patrones se hace necesario diferenciar la escala de los factores ecológicos bajo análisis. Así, a escala geográfica se espera que la estructura taxonómica de la entomofauna de las vegas estudiadas refleje el patrón climático general. Por ejemplo, varias familias encontradas en este trabajo son comunes en humedales de altitudes bajas, de clima templado (Keiper et al., 2002). Sin embargo, factores locales pueden enmascarar el efecto de los factores geográficos como ha sido documentado por Haslett (1997) y Cooper et al. (2010) para otras latitudes. En el área de nuestro estudio, la brusca gradiente altitudinal y la compleja fisiografía de la montaña andina local, con su efecto sobre la redistribución del agua y las características térmicas de sitios particulares (áreas de umbría versus áreas soleadas), generan una heterogeneidad espacial que probablemente afecta la estructura del ensamble de Diptera. Se espera un papel similar de las características fisiográficas de la vega, como su composición florística, tamaño y complejidad estructural, particularmente la presencia de cuerpos de agua o sitios inundados y del clima local semiárido, mediante sus ciclos de humedad-sequedad. A esta situación se agrega el hecho de que el agua presente en las vegas pasa por sustratos mineralógicos que determinan las características químicas del microhábitat acuático o semiacuático de la vega, con posibles efectos sobre las características de la fauna asociada a estos sistemas (Alvial et al., 2012, 2013, 2014; Cepeda-Pizarro et al., 2013).

Este estudio ha aportado algunos antecedentes que documentan el potencial papel de los dípteros como indicadores del cambio ecosistémico del prado húmedo altoandino del desierto transicional de Chile; no obstante, por ahora se carece de antecedentes para proponer familias o grupos de familias, especies o géneros incluidos, que den cuenta de estos cambios.

\section{Conclusiones}

Se registró la presencia de 29 familias de Diptera, registro más alto al encontrado en humedales costeros del centro-sur de Chile; lo que estaría reflejando el mayor grado de pristinidad y disponibilidad de microhábitats de las vegas andinas estudiadas en 
este trabajo. La presencia de grupos asociados al ambiente terrestre refleja la interacción entre la vega y el entorno estepario circundante. Cuatro familias (i.e. Muscidae, Chironomidae, Sphaeroceridae y Heleomyzidae) dominaron numéricamente el ensamble. La riqueza-F estimada de Diptera fluctuó entre 29 y 31 . Se detectó un efecto de la condición hídrica de la vega sobre las relaciones de abundancia entre familias y algunos de los parámetros comunitarios estudiados (e.g. diversidad y disimilaridad). Los índices usados para estimar la equitabilidad del ensamble entregaron resultados no concluyentes.

\section{Agradecimientos}

Esta publicación fue financiada por la Dirección de Investigación de la Universidad de La Serena por medio los proyectos PR14121 (J.C.P.) y DIULS PR15121 (J.P.A.).

\section{Literatura Citada}

Acosta, R.; Prat, N.

2010. Chironomid assemblages in high altitude streams of the Andean region of Peru. Fundamental and Applied Limnology, 177: 57-79.

Ahumada, M.; Faúndez, L.

2007. Manual de reconocimiento de especies de flora de las veranadas: Región de Coquimbo. Servicio Agrícola y Ganadero, División de Protección de Recursos Naturales Renovables, Coquimbo, Chile. 146 pp.

Alvial, I.E.; Tapia, D.H.; Castro, M.J.; Durán, B.C.; Verdugo C.A.

2012. Analysis of benthic macroinvertebrates and biotic indices to evaluate water quality in rivers impacted by mining activities in northern Chile. Knowledge and Management of Aquatic Ecosystems, 407: 01.

Alvial, I.E.; Orth, K.; Durán, B.C.; Álvarez, E.; Squeo, F.A.

2013. Importance of geochemical factors in determining distribution patterns of aquatic invertebrates in mountain streams south of the Atacama Desert, Chile. Hydrobiologia, 709: 11-25.

Alvial, I.E.; Verdugo, C.; Squeo, F.A.; Tapia. D.

2014. Ecology and Environmental Issues of Aquatic Invertebrates in High Mountains Rivers of Northern Chile. En: Goncalves, A.J.B.; Vieira, A.A.B. (eds.). Mountains: Geology, Topography and Environmental Concerns. Nova Science Publishers, Inc, Editors, New York, USA, pp. 229-249.

Anderson, M.J.

2005. Permanova Permutational multivariate analysis of variance. A computer program. Department of Statitics. University of Auckland. New Zeland. 24 pp.

Batzer, D.; Wissinger, S.A.

1996. Ecology of insect communities in nontidal wetlands. Annual Review of Entomology, 41: 75-100.

Beltrán-Concha, M.; Muñoz-Ramírez, C.; Ibarra, J.; Habit, E. 2012. Análisis de la dieta de Diplomystes (Siluriformes: Diplomystidae) de Chile. Gayana, 76: 102-111.

Boggero, A.; Fureder, L.; Lencioni, V.; Simcic, T.; Thaler, B.;

Ferrarese, U.; Lotter, A.; Ettinger, R.

2006. Littoral chironomid communities of Alpine lakes in relation to environmental factors. Hydrobiologia 562: 145-165.

Cepeda-Pizarro, J.; Armijo, A.A.

2014. Dinámica fluviométrica y limnológica de un cuerpo superficial de agua asociado a un prado húmedo de los Andes del norte-centro de Chile. Idesia, 32: 11-19.
Cepeda-Pizarro, J.; Pola, M.

2013. Relaciones de abundancia de hexápodos terrestres en vegas altoandinas del desierto transicional de Chile. Idesia, 31: 31-39.

Cepeda-Pizarro, J.; Pola, M.; Zuleta, C.; González, C.R. 2006. Relaciones de abundancia y diversidad de la entomofauna del humedal Tambo-Puquíos. En Cepeda-Pizarro, J. (ed.). Geoecología de los Andes Desérticos: La Alta Montaña del Valle del Elqui. Ediciones Universidad de La Serena, La Serena, Chile, pp. 475-521.

Cepeda-Pizarro, J.; González, C.R.; Zuleta, C.; Pizarro-Araya, J. 2013. Comparación de la eficiencia de trampas Barber y Malaise para el estudio de la biodiversidad de Hexapoda de vegas altoandinas. Idesia 31: 103-109.

Cooper, D.J.; Wolf, E.C.; Colson, C.; Vering, W.; Granda, A.; Meyer, $\mathrm{M}$.

2010. Alpine Peatlands of the Andes, Cajamarca, Peru. Arctic, Antarctic, and Alpine Research, 42: 19-33.

Figueroa, R.; Palma, A.; Ruiz, V.; Niell, X.

2007. Análisis comparativo de índices bióticos utilizados en la evaluación de la calidad de las aguas en un río mediterráneo de Chile: Río Chillán, VIII Región. Revista Chilena de Historia Natural, 80: 225-242.

Figueroa, R.; Ruiz, V.; Niell, X.; Araya, E.; Palma, A. 2006. Invertebrate colonization patterns in a Mediterranean Chile stream. Hydrobiologia, 571: 409-417.

Figueroa, R.; Sánchez, M.L.; Andreu, A.; Ruiz, V.H.; VidalAbarca, M.R.

2009. Caracterización ecológica de humedales de la zona semiárida en Chile Central. Gayana, 73: 76-94.

Füderer, L.; Ettinger, R.; Boggero, A.; Thaler, B.; Thies, H.

2006. Macroinvertebrate diversity in Alpine lakes: effects of altitude and catchment properties. Hydrobiologia, 562: 123-144.

González, C.R.

2002. The immature stages of two species of Dasybasis from the southern neotropical region (Diptera: Tabanidae: Diachlorini). Annales Zoologici, 52: 271-277.

González, C.R.

2004. The immature stages of Dasybasis pruinivitta (Kröber), from Central Chile (Diptera: Tabanidae). Zootaxa, 573: 1-7.

González, C.R.

2014. Two new species of the genus Dasybasis Macquart, 1847, from Chile (Diptera: Tabanidae: Diachlorini). Zootaxa, 3893: 293-300. 
Grillet, M.E.; Barrera, R.

1997. Spatial and temporal abundance, substrate partitioning and species co-occurrence in a guild of Neotropical blackflies (Diptera: Simuliidae). Hydrobiologia, 345: 197-208.

Haslett, J.R.

1997. Insect communities and the spatial complexity of mountain hábitats. Global Ecology \& Biogeography Letters, 6: 49-56.

Henderson, P.; Seaby, R.

2008. A Practical Handbook for Multivariate Methods. Pisces Conservation IRC House, The Square, Pennington, Lymington, Hants, UK. 224 pp.

Hermosilla, W.; Murúa, R.; Barrios, M.

1975. Estudios ecológicos en cordillera Pelada (provincia de Valdivia) Chile. IV. Distribución estacional de los invertebrados epigeos en turberas. Medio Ambiente, 1: 14-28.

Hinden, H.; Oertli, B.; Menetrey, N.; Sager, L.; Lachavanne, J.B. 2005. Alpine pond biodiversity: what are the related environmental variables?. Aquatic Conservation: Marine and Freshwater Ecosystems, 15: 613-624.

Keiper, J.B.; Walton, W.E.; Foote, B.A.

2002. Biology and ecology of higher Diptera from freshwater wetlands. Annual Review of Entomology, 47: 207-232.

Lods-Crozet, B.; Castella, E.

2009. Colonization by midges (Chironomidae, Diptera) of recently created shallow ponds: implications for the restoration of lacustrine fringing wetlands. Annales de Limnologie International Journal of Limnology, 45: 257-266.

McAlpine, J.F.; Peterson, B.V.; Shewell, G.E.; Teskey, H.J.;

Vockeroth, J.R.; Wood, D.M. (eds.). 1981. Manual of Neartic Diptera. Monograph 27 (Vol. 1). Research Branch, Agriculture Canada. Canada. 674 pp.

McAlpine, J.F.; Peterson, B.V.; Shewell, G.E.; Teskey, H.J.;

Vockeroth J.R.; Wood, D.M. (eds.).

1987. Manual of Neartic Diptera. Monograph 28 (Vol. 2). Research Branch, Agriculture Canada, Canada. 1332 pp.

McElligott, P.E.K.; Lewis, D.J.

1996. Distribution and abundance of immature tabanidae (Diptera) in a subarctic Labrador peatland. Canadian Journal of Zoology, 74: 1364-1369.

Osorio, R.; Cabezas, R.; Reyes, H.; Álvarez, P.; Koné, T. 2006. Humedales altoandinos de la IV Región. En CepedaPizarro, J. (ed.). Geoecología de los Andes Desérticos: La Alta Montaña del Valle del Elqui. Ediciones Universidad de La Serena, La Serena, Chile, pp. 152-239.

Sánchez, D.; Amat-García, G.D.

2005. Diversidad de la fauna de artrópodos terrestres en el humedal Jaboque, Bogotá-Colombia. Caldasia, 27: 311-329.

Sanford, M.R.; Keiper, J.B.; Walton, W.E.

2003. The impact of wetland vegetation drying time on abundance of mosquitoes and other invertebrates. Journal of the American Mosquito Control Association, 19: 361-366.
Sanhueza, D.; Miranda, M.; Gómez, M.; Bonacic, C. 2009. Species richness, diversity and human activities in an elevation gradient of a high-ecosystem in Lagunas Huascoaltinas, Atacama Region, Chile. Ciencia e Investigación Agraria, 36: 411-423.

Scheibler, E.E.; Roig-Junent, S.A.; Claps, M.C.

2014. Chironomid (Insecta: Diptera) assemblages along an Andean altitudinal gradient. Aquatic Biology, 20: 169-184.

Seaby, R.M.; Henderson, P.A.

2006. Species Diversity and Richness Version 4. Pisces Conservation Ltd., Lymington, England. 84 pp.

Squeo, F.A.; Osorio, R.; Arancio, G.

1994. Flora de los Andes de Coquimbo: Cordillera de Doña Ana. Ediciones Universidad de La Serena, La Serena. 176 pp.

Squeo, F.A.; Warner, B.G.; Aravena R.; Espinoza, D.

2006a. Bofedales: high altitude peatlands of the central Andes. Revista Chilena de Historia Natural, 79: 245-255.

Squeo, F.A.; Ibacache, E.; Warner, B.; Espinoza, D.; Aravena, R.; Gutiérrez, J.R.

2006b. Productividad y diversidad florística de la vega Tambo, Cordillera de Doña Ana. En Cepeda-Pizarro, J. (ed.). Geoecología de los Andes Desérticos: La Alta Montaña del Valle del Elqui. Ediciones Universidad de La Serena, La Serena, Chile, pp. 325-351.

Timms, L.L.; Bowden, J.J.; Summerville K.S.; Buddle, C.M. 2014. Does species-level resolution matter?. Taxonomic sufficiency in terrestrial arthropod biodiversity studies. Insect Conservation and Diversity, 6: 453-462.

Trivelli, M.; Huerta, J.

2014. Alcances sobre flora y vegetación de la cordillera de los Andes, Región de Antofagasta. Primera Edición. Ministerio de Agricultura. Servicio Agrícola y Ganadero, Santiago, Chile. 319 pp.

Van der Valk A.G.

2006. The biology of freswwater wetlands. Oxford University Press Inc. New York. USA. 173 pp.

Villagrán-Mella, R.; Aguayo, M.; Parra, L.E.; González, A.

2006. Relación entre características del hábitat y estructura del ensamble de insectos en humedales palustres urbanos del centro-sur de Chile. Revista Chilena de Historia Natural, 79: 195-211.

Wissinger, S.A.; Brown W.S.; Jannot, J.E.

2003. Caddisfly life histories along permanent gradients in high-altitude wetlands in Colorado (USA). Freshwater Biology, 48: 255-270.

Zavala, $\mathrm{H}$.

2006. Hidrología del humedal Tambo-Puquios. En: CepedaPizarro, J. (ed.). Geoecología de los Andes Desérticos: La Alta Montaña del Valle del Elqui. Ediciones Universidad de La Serena, La Serena, Chile, pp. 287-321. 
Anexo Tabla 1. Algunos antecedentes de historia natural de familias de Diptera registradas en vegas de los Andes desértico-transicionales de Chile.

\begin{tabular}{|c|c|}
\hline Familia & Comentarios \\
\hline Agromyzidae & Terrestres, fitófagos. Su presencia en las vegas representa invasiones desde la vegetación zonal \\
\hline Asilidae & Terrestres. Su presencia en las vegas representa invasiones desde la vegetación zonal \\
\hline Bibionidae & $\begin{array}{l}\text { Terrestres. Sus larvas viven en sustratos ricos en humus, hojas en descomposición, entre las } \\
\text { raíces del pasto }\end{array}$ \\
\hline Bombyliidae & $\begin{array}{l}\text { Parásito de una amplia variedad de órdenes de Insecta. Su presencia en las vegas representa } \\
\text { invasiones desde la vegetación zonal }\end{array}$ \\
\hline Calliphoridae & $\begin{array}{l}\text { Terrestres. Las larvas son básicamente coprófagas o carroñeras. Su presencia en las vegas } \\
\text { representa invasiones desde la vegetación zonal }\end{array}$ \\
\hline Ceratopogonidae & $\begin{array}{l}\text { La mayoría de las especies requieren de hábitats con elevada humedad. Una subfamilia } \\
\text { (Leptoconopinae) es de ambientes terrestres (e.g. Leptoconops chilensis descrita para la zona } \\
\text { de Arica) }\end{array}$ \\
\hline Culicidae & Restringida a ambientes lénticos \\
\hline Chironomidae & $\begin{array}{l}\text { La mayoría de las especies requiere de hábitats acuáticos, algunos géneros encontrados en } \\
\text { ambientes semiacuáticos }\end{array}$ \\
\hline Chloropidae & $\begin{array}{l}\text { Terrestres. Poseen larvas fitófagas y saprófagas que pueden alimentarse de algún tipo de recurso } \\
\text { disponible en las vegas }\end{array}$ \\
\hline Dolichopodidae & $\begin{array}{l}\text { Adultos asociados a ambientes terrestres húmedos o bordes de playa, depredan sobre insectos } \\
\text { pequeños y blandos. El hábitat larval es variado, incluyendo hábitats acuáticos y semiacuáticos }\end{array}$ \\
\hline Empididae & $\begin{array}{l}\text { La mayoría de los géneros conocidos es de ambientes terrestres. Solo algunos pocos géneros } \\
\text { están asociados a ambientes semiacuáticos, sus larvas depredan sobre larvas de Simuliidae y } \\
\text { pupas de Trichoptera }\end{array}$ \\
\hline Ephydridae & $\begin{array}{l}\text { Encontrados en ambientes acuáticos y semiacuáticos. Se alimentan de algas, excrementos y } \\
\text { materia orgánica en descomposición. Algunos depredan sobre insectos pequeños }\end{array}$ \\
\hline Heleomyzidae & $\begin{array}{l}\text { Terrestres. Sus larvas son carroñeras, se encuentran en diversos tipos de materia vegetal y } \\
\text { animal en descomposición }\end{array}$ \\
\hline Muscidae & Terrestres. Su presencia en las vegas representa invasiones desde la vegetación zonal \\
\hline Nemestrinidae & $\begin{array}{l}\text { Terrestres. Parásitos de Orthoptera y Coleoptera. Su presencia en las vegas representa invasiones } \\
\text { desde la vegetación zonal }\end{array}$ \\
\hline Otitidae (Ulidiidae) & $\begin{array}{l}\text { Asociados al follaje de vegetación de sitios húmedos. La larva puede encontrarse en materia } \\
\text { vegetal viva o en descomposición y excrementos }\end{array}$ \\
\hline Phoridae & Terrestres. Sus larvas se alimentan de plantas y animales en descomposición y de hongos \\
\hline Psychodidae & $\begin{array}{l}\text { La mayoría de las especies vive en ambientes acuáticos o semiacuáticos, solo una subfamilia } \\
\text { (Phlebotominae) posee representantes terrestres }\end{array}$ \\
\hline Rhinophoridae & $\begin{array}{l}\text { Las larvas son parásitos internos de Isopoda. Su presencia en las vegas representa invasiones } \\
\text { desde el sector zonal }\end{array}$ \\
\hline Sarcophagidae & Su presencia en las vegas representa invasiones desde el sector zonal. \\
\hline Sciaridae & Terrestres, insectos fitófagos, se alimentan de materia vegetal en descomposición y hongos \\
\hline Simuliidae & Restringida a ambientes lóticos \\
\hline Sphaeroceridae & $\begin{array}{l}\text { Terrestres. Larvas se alimentan de estiércol; sin embargo, pueden encontrarse asociadas a todo } \\
\text { tipo de materia orgánica en descomposición }\end{array}$ \\
\hline Syrphidae & $\begin{array}{l}\text { Los adultos se encuentran generalmente sobre flores o revoloteando en lugares soleados. Larvas } \\
\text { se encuentran en un amplio rango de hábitats, tanto terrestres como acuáticos }\end{array}$ \\
\hline Stratiomyidae & Familia primariamente terrestre, pero con representantes de ambientes acuáticos y semiacuáticos \\
\hline Tabanidae & $\begin{array}{l}\text { Adultos asociados a ambientes húmedos, pantanosos. Las larvas depredan sobre diversos } \\
\text { invertebrados, frecuentemente se encuentran en pozas poco profundas, barro y vegetación en } \\
\text { descomposición }\end{array}$ \\
\hline Tachinidae & $\begin{array}{l}\text { Terrestres. Parásitos de una amplia variedad de órdenes de Insecta. Su presencia en las vegas } \\
\text { representa invasiones desde el sector zonal }\end{array}$ \\
\hline Therevidae & Las larvas se encuentran en suelo o materia vegetal en descomposición \\
\hline Tipulidae & Se encuentran en una amplia variedad de hábitats tanto acuáticos y semiacuáticos como terrestres \\
\hline
\end{tabular}

